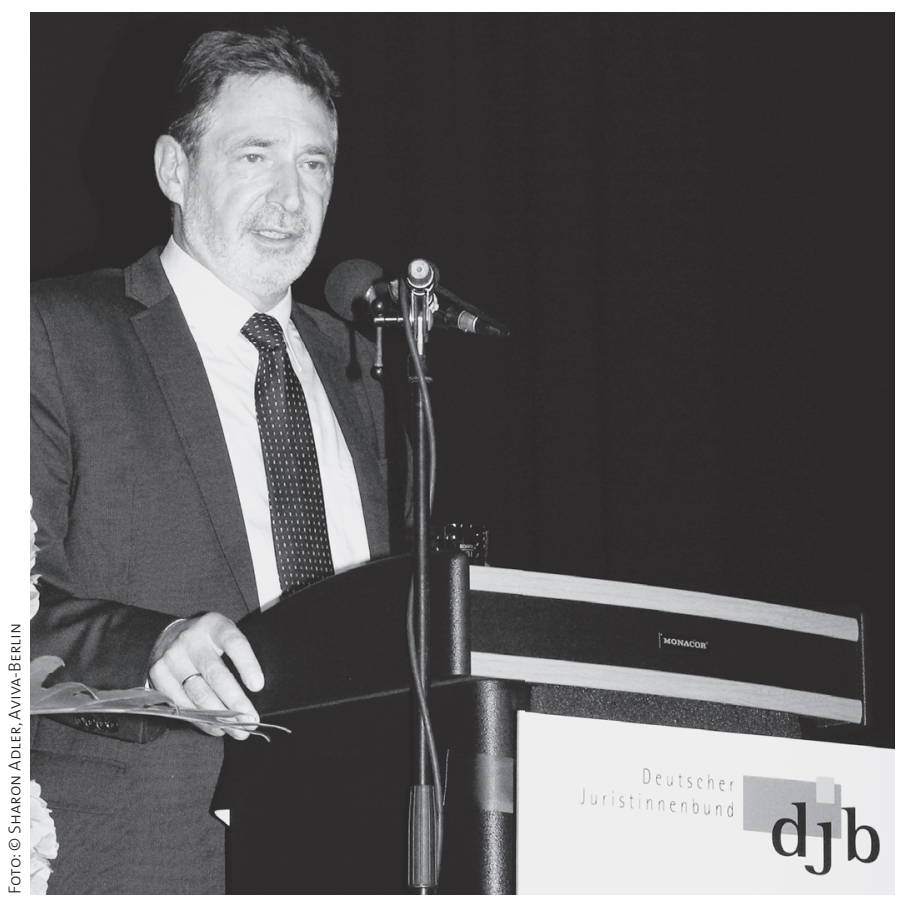

A Oberbürgermeister Jann Jakobs spricht ein Grußwort.

Jahr. Ich habe es selbst kaum glauben wollen, aber jährlich kommen 18 Millionen Tagesbesucher in die Stadt. Die machen das natürlich so, dass sie vorwiegend ein paar Tage in Berlin sind und dann nach Potsdam kommen. Wir arbeiten daran, dieses Verhältnis umzukehren. Sie machen es genau richtig, wie ich finde. Sie tagen in Potsdam, Sie übernachten auch hier, vielleicht fahren Sie am Sonntagnachmittag nach Berlin. Ich finde das vollkommen in Ordnung. Wissen Sie, es ist auch so, dass statistisch zum Ausdruck gebracht wird, dass jede Besucherin und jeder Besucher im Schnitt 30 Euro pro Tag hierlässt und da sind die Unterbringungskosten nicht mit eingerechnet. Ich sage das nur, meine Damen und Herren, damit Sie eine Anregung erhalten hinsichtlich der Ausgaben, die Sie in den nächsten Tagen hier zu tätigen haben. Das ist eine wichtige Einnahmequelle, aber es wäre auch wiederum zu kurz gegriffen, wollte man Potsdam nur auf seine Sehenswürdigkeiten reduzieren. Zugegebenermaßen ist die Stadt kein traditionsreicher Gerichtsstandort, aber sie ist ein wichtiger Gerichtsstandort hier in Brandenburg mit Verfassungsgericht,
Landgericht, Landesarbeitsgericht. Alle genannten Gerichte befinden sich in der Landeshauptstadt Potsdam. Ich lade einmal im Jahr auch die Gerichtspräsidenten zu einem Austausch ein, um darüber zu sprechen und konkret zu verabreden, was hier auf kommunalpolitischer Ebene für die Gerichte auf den Weg gebracht werden soll.

Herr Staatssekretär, ich habe mich natürlich auch gefragt bei dem Thema „Gleichberechtigung“, wie es denn eigentlich in meiner Verwaltung aussieht und ich beginne auch „oben“. Also, der Oberbürgermeister ist ein Mann, das sehen Sie, meine Damen und Herren. Dann kommt die sogenannte Beigeordnetenriege, da sind wir paritätisch besetzt, zwei Frauen und zwei Männer. Das Verhältnis hat sich aber zulasten der Frauen verschlechtert, denn bis vor Kurzem waren es drei Frauen und ein Mann. Dann geht es weiter mit den Leitungsebenen. Mir wurde aufgeschrieben, dass 8,1 Prozent der Männer Leitungspositionen inne haben und nur 7,1 der Frauen. Man könnte nun sagen, naja, da sind wir auf dem Weg. Aber nein, die Situation ist vielmehr so, dass erstens dieser Zustand seit Jahren stagniert und zum zweiten muss man diese Zahl wiederum in Relation zu den Beschäftigten der Stadtverwaltung setzen. Wir haben unter den Beschäftigten zwei Drittel Frauen und ein Drittel Männer. Insoweit haben wir in der Tat noch Nachholbedarf. Es gibt einen Gleichstellungsplan der Landeshauptstadt Potsdam und wir arbeiten emsig daran, dieses Verhältnis zu verbessern. Die meisten Beschäftigen des öffentlichen Dienstes dürfte es in den Kommunalverwaltungen geben. Daher sind sie ein ganz wichtiger Schlüssel, um dafür Sorge zu tragen, dass Frauen in Leitungspositionen kommen. Es gibt hier in den nächsten Jahren sicherlich viel zu tun.

Um über die richtigen Strategien nachzudenken, ist Potsdam der richtige Ort. Dazu wünsche ich Ihnen, meine sehr verehrten Damen, sehr viel Freude, eine anregende Umgebung und viel Biss und Durchsetzungskraft, um Ihre Ziele in den nächsten Jahren durchzusetzen. Potsdam ist ja für das eine oder andere Grundsatzabkommen durchaus bekannt, nicht immer in positiver Hinsicht, aber es könnte ja auch ein Potsdamer Abkommen zur Gleichberechtigung in den öffentlichen Verwaltungen geben. Damit würden wir gerne in die Geschichte eingehen. In diesem Sinne wünsche ich Ihnen viel Freude, viel Spaß und kommen Sie gerne wieder her. Vielen Dank!

\title{
Menschenwürde, Freiheit, Gleichheit
}

\section{Festvortrag für den 39. Jahreskongress des Deutschen Juristinnenbundes $2011^{1}$}

\section{Prof. Dr. Susanne Baer, LL.M. \\ Richterin des Bundesverfassungsgerichts, Karlsruhe}

Mit dem Deutschen Juristinnenbund ist das so eine Sache. Er kommt als ganz harmlose berufsständische Vereinigung daher, nimmt dann aber in einer Vielzahl von Rechtsfragen eminent politisch Stellung. Er wirkt so ganz und gar bodenständig, bürgerlich, macht dann aber Aktionen, die sogenannte bürgerliche Kreise durchaus irritieren. Dazu gehören sicherlich diese anstrengenden Fragen auf den Versammlungen der Aktiengesellschaften: Warum sitzt da keine Frau? Wie ist das mit den

\footnotetext{
1 Die Vertragsfassung wurde beibehalten. Ich bedanke mich für die Recherchen bei Marieke Michel.
} 
Wahlvorschlägen? Was haben Sie getan, um an der Einseitigkeit reiner Männergremien etwas zu ändern? Das wird ganz adrett vorgetragen, ist aber dann doch eine Irritation.

Auf diesem Kongress befasst sich der Deutsche Juristinnenbund mit der gläsernen Decke. Auch das klingt politisch eher harmlos, nämlich nach der eigenen Karriere. Sind wir hier also auf einer Coaching-Veranstaltung? Ist Gleichstellung heute die Frage danach, wer fit genug ist oder wird, um nach oben zu kommen?

Die Antwort lautet: Nein.

Der djb thematisiert das wie immer auf diese ganz besondere, subtile, lächelnd radikale Art. Es ist mit dem djb und auch mit diversen anderen Dingen und Menschen eben so eine Sache. Und darüber will ich sprechen.

\section{Back to basics}

Diese ganz besondere, subtile, lächelnd radikale Art in der Thematisierung von Gleichstellungsfragen hat einen ganz bestimmten, nicht zuletzt grundrechtlich gesicherten Hintergrund. Es ist insofern auch so eine Sache mit der Gleichheit. Sie funktioniert auch grundrechtlich nur, wenn sie ernst und substanziell gemeint ist. Nur ein ganz bestimmtes Verständnis von Gleichstellung, von Gleichheit als Garant menschenwürdiger Freiheit, kann produktive Diskussionen nicht nur, aber auch über Karrieren inspirieren. Es ist dieses Verständnis, dem sich der djb verschrieben hat und das auch im Grundgesetz verankert ist.

Heute droht jedoch allerorten die Gefahr, das aus den Augen zu verlieren. Was ist denn genau gemeint, wenn allerorten von Gleichstellung gesprochen wird? Was kann gemeint sein, wenn alle das gut finden, wofür noch vor ein paar Jahren bestenfalls mitleidiges Lächeln, häufiger aber aggressiver Ärger kassiert wurde: der Ruf nach der Quote? Was ist das, dem sich so viele jetzt zumindest flexibel verschreiben wollen?

Es ist so eine Sache mit der Gleichheit, wenn sie derart plausibel daherkommt. Sie muss im Detail konkretisiert werden. Sie braucht Substanz. Und es ist so eine Sache mit dem Ruf nach der Quote, weil dahinter ursprünglich substanzielle und strukturverändernde, heute aber oft plausible, aber auch flache Deutungen von Gleichheit als Angleichung stehen. Letztere müssen als untauglich und auch als gefährlich entlarvt werden.

Diese gefährlichen Variationen auf die Gleichheit resultieren nicht zuletzt aus der Ambivalenz des Erfolges. Langjährige und mühevolle Kämpfe haben dazu geführt, dass heute alle über die Gleichheit reden. Wir auch. Aber dieses Reden darf nicht zum Kollateralschaden werden.

\section{Alle reden von Gleichheit. Wir auch. Aber anders, ernsthaft, substanziell.}

Allerdings ist es ungemein verführerisch, sich einlullen zu lassen. Wir könnten uns lächelnd zurücklehnen, weil in der Politik, der Justiz, der Wissenschaft, den Unternehmen, den Medien ja nun von Gleichheit die Rede ist und sogar die Frauen erwähnt werden. (Nur in juristischen Texten hält sich der Klä- ger und der Richter und der Arbeitnehmer und der Gesellschafter und der Mandant ziemlich beharrlich - da ist noch Kreativität gefragt.) Wir könnten lächelnd ein Nickerchen machen, wenn heute gängige Gleichstellungsrhetoriken zu Gehör gebracht werden. Es wäre auch so angenehm entspannt, nicht immer diese Fragen zu stellen, auf die Leute dann so genervt reagieren, nicht die Spielverderberin zu sein, endlich einmal ganz selbstverständlich auf der Seite der Gewinner zu stehen und nur noch Witze zu hören, die wirklich witzig sind, sich also im Einverständnis auszuruhen.

Aber so funktioniert das eben nicht. Alle reden von Gleichheit. Wir auch. Aber anders.

Der Hintergrund für die Arbeit des djb ist keine flache, rhetorische Floskel. Es ist kein formelles Verständnis von abstrakter Chancengleichheit, das hier grundlegend wirkt. Vielmehr ist es ein Verständnis von Gleichheit als Grundrecht gegen Diskriminierung, als Grenze einer folglich nicht grenzenlosen Freiheit, als Garantie für ein sozial verkraftbares Höchstmaß an Selbstbestimmung, in Achtung und Anerkennung aller gleichermaßen, in ihrer ganz konkreten Menschenwürde.

Es ist also zur Vermeidung von Kollateralschäden und zur Aufklärung der nur blendenden Rhetoriker ratsam, sich dieser Quellen des eigenen Tuns zu versichern. Es gilt, an die besondere Qualität der Grundlagen zu erinnern. Das bedeutet: Back to basics. Und in der juristischen Variante heißt das: Immer wieder zurück zu den Grund- und Menschenrechten. ${ }^{2}$ Auf diese besondere, subtile, lächelnd radikale Art.

\section{Richterliche Zurückhaltung?}

Dabei stellt sich nun für mich ein kleines Problem. Es ist eigentlich sogar eine ziemlich komplizierte Frage und es ist eine Tugend, was es nicht leichter macht. Sie nennt sich richterliche Zurückhaltung.

Vor einem Jahr hätte ich hier als Wissenschaftlerin gesprochen, sie also mit den neuesten Erkenntnissen der Gender Studies vertraut gemacht. Vermutlich wäre es um Männlichkeitsvorstellungen gegangen, die immer in der Krise sind, aber derzeit auch spezifisch umkämpft. ${ }^{3}$ Ich hätte nicht zuletzt rechtspolitisch Position bezogen. Aber das ist heute anders. Ich bin zwar - das regelt das Bundesverfassungsgerichtsgesetz ausdrücklich ${ }^{4}$ - weiterhin auch als Professorin tätig. Aber in erster Linie bin ich heute Richterin.

Die richterliche Zurückhaltung schützt mich davor, in künftigen Verfahren vor dem Bundesverfassungsgericht auch

2 Eine Sammlung der heute geltenden Menschenrechtsverträge findet sich auf der Webseite des Deutschen Instituts für Menschenrechte, mit einem Rechercheinstrument unter <http://www.dimr. eu/> (Zugriff: 12.10.2011). Für Gleichstellungsfragen besonders relevant: CEDAW, die Frauenrechtskonvention, CERD, die Antirassismuskonvention, und CRD, die Konvention der Rechte von Behinderten.

3 Dies ist ein Forschungsschwerpunkt von Andrea Maihofer, Universität Basel, die das als Thema des Festvortrags zum Jubiläum 2011 wählte. Vgl. auch Michael Meuser, Geschlecht und Männlichkeit, 3. Aufl. 2010.

4 §3 Abs. 4 BVerfGG lautet: „Mit der richterlichen Tätigkeit ist eine andere berufliche Tätigkeit als die eines Lehrers des Rechts an einer deutschen Hochschule unvereinbar. Die Tätigkeit als Richter des Bundesverfassungsgerichts geht der Tätigkeit als Hochschullehrer vor." 
nur den entfernten Anschein von Befangenheit zu erwecken. Da der Erste Senat, dem ich angehöre, als Grundrechtssenat tendenziell mit allem befasst wird, was Menschen bewegt, und genau das sorgsam hütet, worüber ich hier spreche - Menschenwürde, Freiheit, Gleichheit - spricht also viel dafür, jetzt lieber zu schweigen.

Die richterliche Zurückhaltung dient auch noch grundlegender dazu, das Vertrauen in die Unparteilichkeit der Justiz $\mathrm{zu}$ wahren. Gerade für das Bundesverfassungsgericht ist das extrem wichtig. Gerade ein solches Gericht muss Vertrauen genießen und respektiert werden. Denn wir müssen Entscheidungen verantworten, die sich erforderlichenfalls gegen die politische, also die gewählte Mehrheit oder auch gegen eine gesellschaftliche Mehrheit stellen.

So hat das Bundesverfassungsgericht bekanntlich eine ganze Serie von Entscheidungen zu den Grundrechten transsexueller Menschen getroffen, die den Gesetzgeber - die demokratisch konstituierte Mehrheit - gezwungen haben, zugunsten einer Minderheit tätig zu werden. Die politische und wohl auch die gesellschaftliche Mehrheit musste dabei auch immer Vorstellungen davon ändern, was normal, was Normalität ist. Solche Entscheidungen erschüttern die privilegierte Ruhe der tradierten Selbstverständlichkeiten. Sie liegen nicht im mehrheitlichen, populären Trend. Sie sind für manche unbequem. Sie brechen mit Tabus.

Das gilt nicht nur für die Rechtsprechung zum Grundrechtsschutz von Transsexuellen, sondern auch für Entscheidungen zum Lebenspartnerschaftsrecht oder zum nachehelichen Sorgerecht oder zu den Vätermonaten oder zum Mutterschutz in der Sozialversicherung. Es gilt auch für den Hartz IV-Beschluss zur Neuberechnung der Sozialleistungen. Das Bundesverfassungsgericht müsste sich ja gar keine Gedanken machen, wenn es nicht eben um solche ernsthaften und tief greifenden Konflikte zwischen Mehrheiten und Minderheiten ginge.

Will das Gericht aber mit den Antworten respektiert werden, die es in Anwendung des Grundgesetzes findet und die strukturell eigentlich nie alle glücklich machen können (denn sonst hätte es des Schiedsspruches nicht bedurft), benötigt es eben ein hohes Maß an Vertrauen. Und dieses Vertrauen beruht auch auf richterlicher Unabhängigkeit. Und diese verlangt nach richterlicher Zurückhaltung. $\mathrm{Zu}$ vielen Themen kann und will ich also definitv nichts sagen. Es ist in den nächsten 12 Jahren nicht meine Aufgabe, rechtspolitische Reden zu halten. Worüber soll, kann und will ich also sprechen? Über die gläserne Decke, das Thema des Kongresses?

\section{Schein und Sein}

Da gibt es ein zweites Handicap. Es oszilliert um die Spannung zwischen Schein und Sein. Man weiß zwar nie so genau, warum eine Einladung zu einem Vortrag ausgesprochen wird. Aber es könnte in meinem Fall etwas damit zu tun haben, dass ich Richterin des Bundesverfassungsgerichts bin, daneben noch Professorin an der Humboldt-Universität und der Michigan Law School, einst Vizepräsidentin der Universität, Beraterin der DFG, der Europäischen Kommission, einiger Ministe- rien - also sozial, salopp formuliert, die Angebernummer, strukturell ein Paradefall von Karrierefrau. Es könnte also sein, dass ich hier als Exemplar derjenigen Gattung stehe, die nun auch in die Aufsichtsräte eingelassen werden soll. Ich mag zwar kein Prachtexemplar sein, aber immerhin.

Dann wäre es jedoch (wie beim djb) auch mit mir so eine Sache: Sie kommt recht bürgerlich und ganz karrieremäßig daher ... und ist dann doch irgendwie noch anders. Ich könnte zwar versuchen, das Bild als Vorbild der Karrierefrau mit einer wirklich netten, auch schlauen und an einigen Stellen dezent lächelnd fordernden Rede zu bestätigen. Aber das würde mir selbst und auch der Forderung nach Gleichstellung Unrecht tun.

Was mich selbst betrifft, will ich wohl nicht einfach immer nur nett sein und das auch keinesfalls immer. Vermutlich bin ich auch nicht nur dezent, und da hilft kein Anzug und keine weiße Bluse. Es gibt zudem nicht nur die glorreichen Stationen der Karriere - Professur, Präsidium, Verfassungsgericht -, sondern auch die Stationen autonome Frauenbewegung, Widerstandsaktionen (so hieß das in den Achtzigerjahren!) und feministische - ja: feministische! - Rechtswissenschaft, feministische Rechtspolitik und feministische Wissenschaft und Wissenschaftspolitik. Also: Lila Latzhose unter grauem Kostüm? Außerdem gibt es noch das Leben als lesbische Frau, das ich zwar nie plakatieren wollte, aber doch irgendwann entschieden habe, es nicht zu verstecken. Es haben dann andere plakatiert, und angesichts der erschreckend geringen Zahl ganz selbstverständlich offen lesbisch oder sonst anders als erwartet lebender Frauen in politisch oder juristisch oder sonst öffentlich sichtbaren Positionen geschieht das wohl zu Recht. Wer mich also als Karrierefrau kaufen oder verkaufen will ... das ist so eine Sache.

Es ist genau so eine Sache wie mit dem djb oder wie mit der simpel-salonfähigen Version von Gleichheit und Gleichstellung. Denn wer bei Gleichheit nur an formelle Chancengleichheit denkt, denkt zu kurz. Wer bei Gleichstellung nur noch möglichst abstrakt an Gerechtigkeit appelliert, greift zu kurz. Wer bei Quote und Aufsichtsrat nur an die nett-adretten Karrierefrauen denkt, nicht aber an strukturelle Veränderungen, verflacht die Diskussion. Es geht nicht um eine freundliche Kollegin im Aufsichtsrat, die für die Atmosphäre gut ist, die zwar nicht mehr Kaffee kochen muss, aber doch wirklich nett die Männerrunde schmückt. Die freundliche Kollegin ist zwar tatsächlich ein Geschenk, wenn sie auftaucht. Aber hier geht es um wirkliche Veränderung, nicht um abstrakte Chancen, tatsächlich um Gleichheit als ein substanzielles Recht im Zusammenspiel mit Freiheit und Menschenwürde. Also wieder: back to basics, die Grund- und Menschenrechte. Über diese kann und will ich sprechen.

\section{Grundrechte konkret}

Nun ist es auch so eine Sache mit den Grundrechten. Sie sind knapp gefasst und sollen viel bewirken. Also müssen sie ausgedeutet, müssen konkretisiert werden. Das Bundesverfassungsgericht ist in unserer rechtsstaatlichen Demokratie die Institu- 
tion, die immer wieder amtlich an diese basics erinnert und erinnern soll. Es hütet die Verfassung, konkretisiert also das Grundgesetz anlässlich der Vielzahl von Fragen, die an das Gericht herangetragen werden.

Das Bundesverfassungsgericht muss zugunsten der Demokratie sicherstellen, dass die Spielregeln eingehalten werden. Über große Fragen muss das Parlament entscheiden - das hat das Gericht gerade in Fragen der europäischen Union sehr deutlich betont. Über große Fragen muss im Parlament auch gut informiert befunden werden - das hat das Gericht in der Entscheidung zu den Hartz-IV Sätzen vorgegeben. Und das Gericht schützt die Substanz der Grundrechte - auch das ist Teil des Beschlusses zu Hartz IV: Die Menschenwürde ist kein Abstraktum, sondern verlangt vom Gesetzgeber, wirklich hinzuschauen, was würdiges Leben in Deutschland heute bedeutet.

Grundrechtsschutz bedeutet also nicht nur, der Staatsgewalt Einhalt zu gebieten, wenn sie die Freiheit mit Füßen tritt. Das ist der Klassiker. Es bedeutet auch, gewählte Mehrheiten zu stoppen, wenn sie zu weit gehen, oder demokratisch gewählte Mehrheiten anzustoßen, wenn sie etwas unterlassen, was zum Schutz aller Grundrechte zu tun ist.

Grundrechtsschutz bedeutet damit immer wieder, große Worte auch gegen die verflachenden Deutungen zu verteidigen, die eine Mehrheit für überzeugend hält. Denn Mehrheiten tendieren nach ihrer inneren Logik dazu, die Interessen von Minderheiten zu übergehen. Die innere Logik der Grundrechte ist genau deshalb der Minderheitenschutz. Und denken Sie bei Minderheit bitte auch, aber nicht nur an die Sorben in Brandenburg oder an religiöse oder ethnisierte oder andere Minderheiten. Hier geht es ohnehin nicht um Essentialisierung, um Gruppismus. Denken Sie an sich selbst: Ihr Alter, Ihre Herkunft, Ihre derzeitige Lebenssituation - manches wird zu Zeiten anerkannt, anderes verkannt, manches ist zu Zeiten Mainstream, anderes nicht. Den Grundrechten geht es um Minderheitsschutz in diesem politischen Sinn; sie schützen Aspekte eines selbstbestimmten Seins, die als anormal ausgegrenzt, in einem umfassenden Sinne „behindert“ werden. „Minderheiten“ sind in der Demokratie alle, die zu einem bestimmten Zeitpunkt nicht regieren, und Minderheitsinteresse ist alles, was bei einer Mehrheit gerade keinen Platz findet. Bestenfalls wechselt das. Daher bauen auch rechtsphilosophische Konzepte zur Legitimation demokratischer Entscheidungen darauf, dass jeder und jede von uns einmal in der Situation der Unterlegenen sein kann, Entscheidungen also nie so fallen dürfen, dass die basics verloren gehen. Um das zu sichern, gibt es die Verfassungsstaatlichkeit, den Konstitutionalismus.

Wer sich im politischen Kampf gegen andere durchsetzt, hat offensichtlich auch nur wenig Grund, die Interessen der Anderen zu wahren. Das ist in Ordnung, denn es gibt große Spielräume und zahlreiche Möglichkeiten, heute Gesellschaft und das Leben in ihr zu gestalten. Doch stößt jeder Gestaltungsspielraum auf eine Grenze, wenn Grundlegendes auf dem Spiel steht. Grundlegend sind eben Menschenwürde,

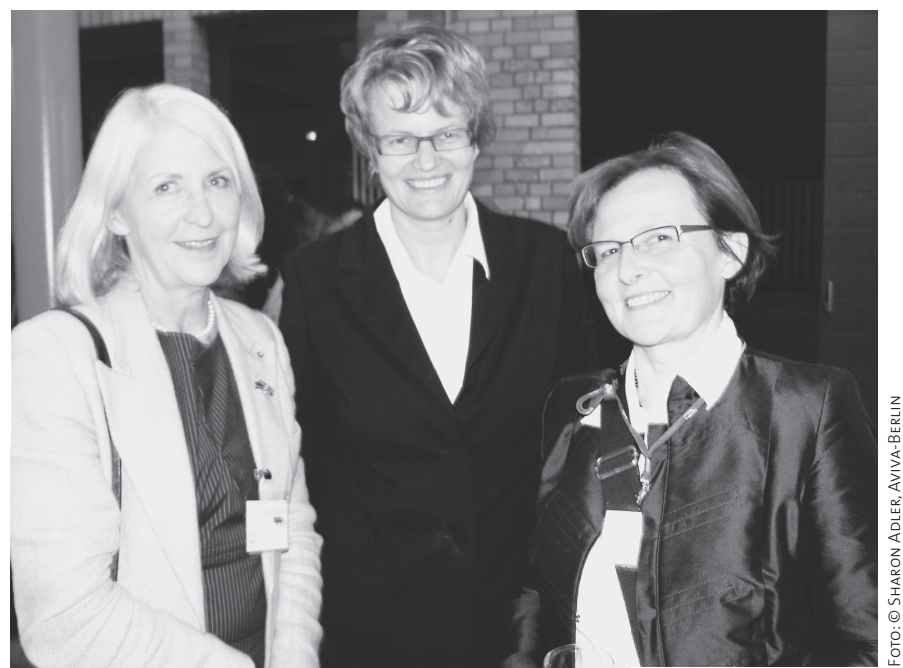

A Rechtsanwältin Margret Diwell (Präsidentin des djb von 2001-2005, Präsidentin des Berliner Verfassungsgerichtshofs), Prof. Dr. Susanne Baer, LL.M. (Richterin des Bundesverfassungsgerichts) und Prof. Dr. Margarete Schuler-Harms (Vorsitzende der Kommission Recht der Sozialen Sicherung im djb) (v.l.n.r.).

Freiheit und Gleichheit. Verfassungsgerichte stoppen also Mehrheiten nicht, weil sie die Dinge anders regeln wollen oder weil irgendein Interesse nachrangig behandelt wird. Verfassungsgerichte müssen eingreifen, wenn sie gerufen werden, weil und wenn Mehrheiten grundlegend zu weit gehen, bei den basics.

\section{Alltagsüberzeugungen und Minderheitenschutz}

Die Klärung dieser basics ist nun besonders schwierig, wenn Mehrheiten Alltagsüberzeugungen aktivieren, also ganz plausibel agieren, im Einklang mit dem Mainstream handeln. Wenn der Gesetzgeber oder auch die Instanzgerichte Grundrechte verkennen, sind das ja selten bewusst geplante Attacken. In der bundesdeutschen Geschichte der Gleichberechtigung im Geschlechterverhältnis hat der Gesetzgeber zwar immer einmal wieder bewusst nichts getan - das Eherecht nicht geändert, das Familienrecht nicht geändert, das Sozialrecht nicht geändert. Es kommt auch heute noch vor, dass klare Vorgaben zum Grundrechtsschutz - auch solche des Europäischen Gerichtshofes oder solche, die sich aus ratifizierten Konventionen über Menschenrechte ergeben - nicht mit der gebotenen Eile und im gebotenen Umfang umgesetzt werden. Das ist bestenfalls den tatsächlichen Herausforderungen des Gesetzgebungsverfahrens geschuldet; der schlechtere Fall der Verweigerung ist selten. Dann regiert das politische Kalkül Karlsruhe möge die Kohlen aus dem populistischen Feuer holen. Ganz selten ist die Renitenz. Besser wäre es in jedem Fall, der Gesetzgeber würde den Mut finden, auch insofern unpopuläre Dinge selbst zu regeln.

Heute geht es jedoch viel häufiger um unbewusste Auslassungen und systemische Verwerfungen in komplizierten Regelungssystemen. Genau diese schleichen sich auch gerne ein, wenn sie sich mit den erwähnten Alltagsüberzeugungen decken. Und sie sind nur zu entlarven, wenn wir uns unserer 
Maßstäbe sicher sind, der basics: Gleichheit, Freiheit und Menschenwürde.

\section{Das Beispiel „Vereinbarkeit“}

Dafür gibt es zahlreiche Beispiele. Nehmen Sie die Diskussion um die „Vereinbarkeit“. Es geht um Fragen, die juristisch das Arbeits- und Sozialrecht, das Ehe- und Familienrecht, das gesamte Wirtschaftsrecht, aber auch das Kommunalrecht oder das Planungsrecht berühren. Es geht um die komplizierte rechtliche Rahmung selbstbestimmter Lebensentwürfe in einer Gesellschaft, die soziale Sicherheit und auch Anerkennung wesentlich an Erwerbsarbeit knüpft.

Also: Vereinbarkeit? Wer davon ausgeht, dass Frauen zuhause bleiben und Männer arbeiten gehen, oder etwas moderner meint, dass auch Frauen arbeiten gehen können, aber dann jemand anders zuhause bleiben muss oder das Zuhause dann woanders liegt, orientiert sich an tradierten Alltagsüberzeugungen - eben: Stereotypen - und behandelt zwei stereotype Lebensentwürfe vielleicht sogar gleich. Und das nennen manche dann Vereinbarkeit. Das klingt plausibel. Aber das verkennt die basics.

In dieser Version geht es nur um die eine Freiheit oder die andere Freiheit - Karriere oder Kinder -, aber nicht um gleiche Anerkennung selbstbestimmter Lebensgestaltung, also um Gleichheit in der Freiheit, zu variieren. Dann geht es nur um zwei konkurrierende Rollenbilder, aber das ernsthafte Versprechen der Gleichheit in einer freiheitlichen Gesellschaft erfüllt sich damit nicht. Es hat ja wenig mit Selbstbestimmung zu tun, wenn wir entscheiden dürften, entweder mit den Kindern zuhause oder aber mit und genau wie die Herren im Aufsichtsrat zu sitzen. Wir sind davon nicht nur weit entfernt. Es genügt auch nicht, weder punktuell noch im Lebensverlauf. Wenn die eine Freiheit in Altersarmut endet und die andere beim Dinner im Adlon - was für eine Freiheit oder Gleichheit ist das?

Die Sachverständigen-Kommission zur Erarbeitung eines Gleichstellungsberichts für die Bundesregierung hat das sehr genau analysiert und folgerichtig kritisiert. ${ }^{5}$ Die Stellungnahme der Bundesregierung dazu lässt den Clou, höflich formuliert, jedoch nicht ganz deutlich werden. Tatsächlich fordert die Kommission eine Abkehr von der Fixierung auf die Wahlfreiheit und eine Politik, die Gestaltungsfreiheit im Rahmen von Gleichstellungspolitk sichert. ${ }^{6}$ Achten Sie auf die Verschiebung oder genauer: auf die Verknüpfung: Gestaltungsfreiheit als Kern der Gleichstellungspolitik. Da sichert Gleichheit Freiheit, so wie die Freiheit davor schützt, im Namen der Gleichheit in Rollen gezwängt zu werden.

\section{Grundrechtsdogmatik}

Das Bundesverfassungsgericht betont dies seit über 20 Jahren. Ich zitiere:

„Der Satz „Männer und Frauen sind gleichberechtigt“ will nicht nur Rechtsnormen beseitigen, die Vor- oder Nachteile an Geschlechtsmerkmale anknüpfen, sondern für die Zukunft die Gleichberechtigung der Geschlechter durchsetzen. Er zielt auf die Angleichung der Lebensverhältnisse. So müssen Frauen die gleichen Erwerbschancen haben wie Männer. Überkommene Rollenverteilungen, die zu einer höheren Belastung oder sonstigen Nachteilen für Frauen führen, dürfen durch staatliche Maßnahmen nicht verfestigt werden. Faktische Nachteile, die typischerweise Frauen treffen, dürfen wegen des Gleichberechtigungsgebots des Art. 3 Abs. 2 GG durch begünstigende Regelungen ausgeglichen werden. “7

Wenn das nicht klare Worte sind! Sie stammen nicht aus einer politischen Erklärung von 2011 - auch da finden sich interessante Punkte ${ }^{8}$-, sondern aus der Entscheidung zum Nachtarbeitsverbot von 1992. Einige dieser Worte stehen heute auch im Grundgesetz, das 1994 (in Art. 3 Abs. 2 Satz 2) nachgebessert wurde. Aber schon damals, 1992, konkretisierte das Gericht, dass es bei der Gleichheit nicht um eine Formalität geht, sondern um etwas Substanzielles.

Damit wird auch die Rolle deutlich, die das Verfassungsgericht in einer Demokratie spielen muss: Staatliche Maßnahmen, also Vorgaben, auf die sich Mehrheiten geeinigt haben, dürfen überkommene Rollenverteilungen, also Muster, die Mehrheiten auch noch gut finden, nicht verfestigen. Das ist die Aufgabe: Mehrheiten notfalls bremsen, Vielfalt sichern. Oder anders: Verbiete niemandem, traditionelle Muster zu leben, aber zwinge auch niemanden dazu! Trage dem Mehrheitswillen Rechnung, schütze aber auch die, die sich ihm nicht unterwerfen, wenn das nicht absolut zwingend ist!

\section{Vielfalt der Lebensentwürfe}

Das Gericht hat diese Grundsätze - echte Gleichheit, wirklich Selbstbestimmung! - vielfach in Anwendung gebracht. Und es hat dabei eben nie nur geklärt, was Gleichheit bedeutet. Es muss immer auch klären, dass Selbstbestimmung Vieles heißen kann, und dass die allen zukommende Menschenwürde dazu auffordert, die Vielfalt der Lebensentwürfe auch anzuerkennen.

Das zeigt auch ein kleiner jüngerer Beschluss des Bundesverfassungsgerichts zum Mutterschutz. ${ }^{9}$ Die Entscheidung, in den

5 Gutachten der Sachverständigenkommission an das Bundesministerium für Familie, Senioren, Frauen und Jugend für den Ersten Gleichstellungsbericht „Neue Wege - Gleiche Chancen Gleichstellung von Frauen und Männern im Lebensverlauf", hg. vom BMFSFJ, Berlin 2011; insbes. S. 91.

6 Stellungnahme der Bundesregierung zum Gutachten der Sachverständigenkommission. Erster Gleichstellungsbericht, hg. vom BMFSFJ 2011.

7 BVerfGE 85, $191<207\rangle$ - Nachtarbeit. Vorläufer war BVerfGE 74, 163 $\langle 180\rangle-$ Witwenrente. Umfassend zur Entwicklung nach wie vor Sacksofsky, U., Das Grundrecht auf Gleichberechtigung, 2. Aufl. 1996; kurz in Foljanty, L./Lembke, U., Feministische Rechtswissenschaft: Ein Studienbuch, 2006.

8 Am 7. März 2011 wurde der Europäische Pakt für die Gleichstellung der Geschlechter von 2006 durch die Ministerinnen und Minister des Rates der Europäischen Union für Beschäftigung, Soziales, Gesundheitswesen und Verbraucherschutz erneuert. Dieser fordert die EU-Mitgliedsländer auf, „Maßnahmen zum Abbau geschlechtsspezifischer Unterschiede und zur Bekämpfung der Geschlechtersegregation auf dem Arbeitsmarkt" zu ergreifen. Weiterhin hat die EU-Kommission am 21. September 2010 eine Fünfjahresstrategie verabschiedet, mit der die Chancengleichheit in Europa vorangetrieben werden soll.

9 BVerfG, 1 BvR 1409/10 vom 28.4.2011, unter <www.bverfg.de〉 (Zugriff: 12.10.2011). 
Mutterschutz zu gehen, ist - zum Schutz der Mutter - keine freie Entscheidung. Deshalb darf sie auch nicht bestraft werden. Ganz im Gegenteil: Wenn der Gesetzgeber Frauen - zum eigenen Schutz - zwingt, rund um die Geburt nicht erwerbstätig zu sein, darf er bei der Berechnung der Rente später nicht sagen, dass das dann aber weniger wert ist als die Zeit im Job.

Warum nicht? Weil es hier keine freie Entscheidung gab, mehr oder weniger zu arbeiten. Weil es um Gleichbehandlung geht für alle, die am Arbeitsplatz aus guten Gründen fehlen. Weil auch das Altern in Würde - und nicht in Altersarmut ein hohes Gut ist.

Das war ein zweites Beispiel, doch finden sich noch viel mehr. Denn es wirkt sich überall aus, wenn wir Gleichheit oder Freiheit oder Würde nicht flach und nicht isoliert voneinander denken, sondern sie als fundamentale Trias der Grundrechte verstehen.

\section{Sexuelle Gewalt vor Gericht}

Deutlich wird das auch beim Strafrecht gegen sexuelle Gewalt. Hier spielen Grund- und Menschenrechte auf vielfache Weise eine oft unterschätzte Rolle. Es geht nicht nur um die Dauer der Verfahren, die als Teil effektiven Rechtsschutzes garantiert ist. Es geht auch um den Umgang mit Menschen vor Gericht, der fair sein muss, also nicht von Stereotypen geleitet sein darf, was sich auf die Wahrnehmung der Sachverhalte ebenso auswirkt wie auf das Prozessrecht, unter anderem in Beweisfragen. ${ }^{10}$

Die Tribunale der Strafverfahren gegen Prominente zeigen jedoch, wie wenig da bislang tatsächlich verändert werden konnte. Die Botschaft lautet erschreckend häufig noch und wieder: Frauen lügen, arme Männer. Oder globalisiert: Amerikaner moralisieren, arme Franzosen. Solche Diskussionen um Vergewaltigung erweisen sich bei genauerer Betrachtung dann allzu oft als Debatten um eine idealisierte und damit auch flache Version von Selbstbestimmung und Freiwilligkeit (die hätte ja nein sagen können!) oder gar Debatten um ebenso flache nationale Klischees (französische Erotik und amerikanische Prüderie). Tatsächlich stellt sich auch hier jedoch die Frage nach Selbstbestimmung im Kontext sexualisierter, geschlechtsspezischer und ethnisierter, sich ökonomisch verfestigender Ungleichheit. Es geht also auch hier um Freiheit im Kontext, um Gleichheit und Würde.

\section{Die Gläserne Decke}

Das gilt auch für Diskussionen zur gläsernen Decke, dem Thema dieses Kongresses. Die Metapher ist ohnehin irreführend: Menschen, die traditionell nicht dazu gehören, stoßen nicht ganz spät an eine gläserne Decke, sondern arbeiten sich durch ein Labyrinth: da stehen immer wieder Mauern im Weg, die umgangen oder überwunden werden müssen. ${ }^{11}$ Allerdings ist in Deutschland vor den Aufsichtsräten und Vorstandspositionen tatsächlich meistens Schluss. Diese letzten Mauern will der djb, will FidAR ${ }^{12}$ durchbrechen. Wie klingt nun diese Debatte, wenn wir uns auf Gleichheit, Freiheit und Menschenwürde besinnen?
Frauen in Aufsichtsräten und Vorständen - das ist als Gleichstellungsvorgabe auch und gerade im Kontext der unternehmerischen Freiheit zu verstehen, also kommen Freiheit und Gleichheit hier gut zusammen. Zudem will bekanntlich keine Frau auf ein Stereotyp Frau reduziert werden, also gehört die Achtung der Individualität dazu, was sich als Menschenwürde bezeichnen lässt. Es geht also auch darum, nicht zur effizienten Maschine zu werden, auch nicht zum Objekt einer Inszenierung, und all das nicht auf Kosten anderer. ${ }^{13}$ Folglich zielt die Forderung nach Gleichstellung hier nicht nur auf Ausnahmefrauen in Führungspositionen. Vielmehr geht es um Unternehmenspolitik und Unternehmenskultur eingedenk eben nicht nur rhetorisch gemeinter Grund- und Menschenrechte: Individualität achten, gleich behandeln und bewerten, Freiheiten austarieren.

Und es ist wunderbar, dass sich Viele zur Aufgabe machen, genau das zu klären.

Das Bundesverfassungsgericht hat gewichtige Worte gefunden und Weichen gestellt, wo es darum ging, der Gleichberechtigung Gehalt und Kontur zu geben. Es betont, dass wir die Freiheit im sozialen Kontext sehen müssen, wo ein strukturelles Ungleichgewicht sogar die Vertragsautonomie beschränken kann. ${ }^{14}$ Es nimmt die Menschenwürde als Schutz vor Prekarisierung ernst. Dies geschieht allerdings nur, wenn das Gericht angerufen wird und wenn Argumente vorgetragen werden, die überzeugen können. Aber das Gericht tritt dann eben auch immer den flachen Versionen von Gleichheit entgegen. Es betont die Gleichheit als substanzielles Recht gegen Diskriminierung und zugunsten der Selbstbestimmung.

Heute geht es eingedenk dieser Grundlagen folglich darum, die Diskussion um die Gläserne Decke nicht auf die Bitte um ein Chancenprogramm für einige wenige angepasste Karrierefrauen zu reduzieren. Denn lassen Sie sich nicht täuschen. Die Politik- und Rechtswissenschaftlerin Sabine Berghahn hat das herausgearbeitet ${ }^{15}$ : Der allfällige Ruf nach der Quote ist heute oft flach oder gefährlich, denn er zieht nicht die Konsequenzen aus dem, was wir bereits an Erfahrungen gesammelt

10 Ein interessantes Beispiel für eine kontextualisierte Betrachtung findet sich im Fall Prosecutor v. Akayesu, Case No. ICTR 96-4-T, Judgment, 688 (Sept. 2, 1998). Zu den Hintergründen MacKinnon, Catharine A., Defining Rape Internationally: A Comment on Akayesu, 44 Columbia J of Transnat. L 940 (2006).

11 Vgl. Eagly, Alice/Carli, Linda, Through the Labyrinth: The Truth About How Women Become Leaders, 2007.

12 Frauen in die Aufsichtsräte e.V. ist die Initiatorin der Kampagne. Sie haben einen „Women-on-Board-Index" erarbeitet. Mehr unter 〈www.fidar.de〉. Zudem veröffentlicht das DIV ein ManagerinnenBarometer, unter $\langle$ www.diw.de $\rangle$. Zahlen finden sich auch beim Statistischen Bundesamt <www.destatis.de〉 (Zugriff: jeweils 12.10.2011).

13 Vgl. Zimmer, Reingard, Mindestarbeitsbedingungen für Frauen weltweit - Sicherung von Mindeststandards für Arbeitsbedingungen von Frauen durch das Recht der Internationalen Arbeitsorganisation?, In: Streit 3/2010, S. 109-118.

14 Das Bundesverfassungsgericht hat mehrfach entschieden, dass Autonomie unter Bedingungen struktureller Ungleichheit als gestörte Parität in Fremdbestimmung umschlagen kann. Vgl. u.a. BVerfGE 81,$242 ; 89,214 ; 103,89$.

15 Vortrag auf der Tagung der deutschsprachigen RechtssoziologieVereinigungen „Der Kampf ums Recht“, Wien 2011. 
haben. Wir wissen eigentlich, wie welche „Quoten“ funktionieren, denn im öffentlichen Dienst gibt es - neben den Quoten der politischen Parteien - seit Langem eine Vielzahl von Fördervorgaben. Im Erwerbsleben haben die eingesetzten Instrumente der Frauenförderung - anders als in Parlamenten bislang jedoch substanziell und nachhaltig sehr wenig bewegt. Dafür gibt es viele Gründe. Nach wie vor hält sich die irrige Behauptung, die Quote sei - nicht zuletzt im Lichte des AGG - rechtswidrig. Das ist nicht nur falsch ${ }^{16}$, sondern oft auch wider besseren Wissens dreist. Zudem wird Frauenförderung noch viel zu selten ernsthaft implementiert, Umgehung also leicht gemacht. Auch läuft die Kontrolle über individuellen Konkurrenzrechtsschutz meist ins Leere (was ein Grund sein muss, im Antidiskriminierungsrecht über kollektiven Rechtsschutz intensiv nachzudenken).

\section{Gleichstellung in der Justiz}

Es ist nun geradezu erschreckend, dass auch in Bereichen, von denen es immer hieß, da seien Frauen jetzt vorn, die Frauen auf Jahre ganz weit weg von den Spitzenpositionen positioniert worden sind. Ein Beispiel dafür ist ausgerechnet die Justiz. Der Parlamentarische Staatssekretär im BMJ, Dr. Stadler, hat die Zahlen bereits genannt ${ }^{17}$ : Die Richterwahlen sind eben solche und weithin keine Wahlen von Richtern und Richterinnen. Gerade die Jahre 2009 und auch 2011 waren, so der Kommentar einer Richterin, „ein Desaster, das aber leider keinen interessiert”. 2009 wurden 14 Positionen am BGH neu besetzt, gewählt wurden 13 Männer und eine Frau. Die Konferenz der Justizministerinnen und Justizminister, die JuMiKo, hat sich im Mai 2011 mehrheitlich für die Einführung einer gesetzlichen Frauenquote in den Führungs- und Kontrollgremien der Wirtschaft ausgesprochen. Ist das, etwas provokant formuliert, substanzielle Gleichheit nur für andere, daheim aber die flache Version?

Der Anteil von Männern und damit auch der Anteil von Frauen an den obersten Gerichten indiziert jedenfalls ein tief greifendes Problem: beim BGH arbeiten zu 79 Prozent Männer, beim BVerwG zu 73 Prozent Männer, beim BFH zu 80 Prozent Männer, beim BAG zu 71 Prozent Männer und nur beim BSG sind einigermaßen paritätisch Männer und Frauen richtend tätig. Was bedeutet das noch? Die Armen, Kranken und Schwachen und die hohen Fallzahlen im Sozialrecht für die Frauen, die harten Fragen der Verwaltung und des Steuerrechts für die Jungs? Und was bedeutet die Situation bei den Vorsitzenden? Beim BGH ist die Zahl der Vorsitzenden Richterinnen im Jahr 2011 von vier auf zwei gesunken. Wenn (nicht nur) ein Gerichtspräsident dann sagt, „die Frauen wollten ja nicht“, rekurriert das auf ein abstraktes, flaches Ideal der Wahlfreiheit. Mit substanzieller, gleicher Freiheit (verfassungsrechtlich formuliert: mit der Sicherung der Bedingungen zur Inanspruchnahme grundrechtlicher Freiheit) hat das nichts zu tun.

Den basics der Grund- und Menschenrechte kämen wir tatsächlich näher, wenn es eine nicht zuletzt grundgesetzkonforme Personalpolitik auch der Bundesländer gäbe. Das be- deutet in der Praxis eine frühzeitige Förderung nicht nur auf der Ebene der Oberlandesgerichte, sondern auch bei den Amtsgerichten und Landgerichten. Das bedeutet auch, die derzeitigen Quoten zumindest zu relativieren. Bei der Wahl an die Bundesgerichte gibt es faktisch Quoten zugunsten der Bundesländer und zugunsten der Parteifreunde. Das sieht das Grundgesetz übrigens nicht vor. Die Grundrechte gebieten und die Justiz braucht - ernsthafte, leistungsgerechte und für die Qualität der Rechtsprechung funktionale Fairness. Die Besinnung auf die basics tut also not. Auch vor Ort, vor der eigenen Haustür - in der eigenen Kanzlei, am eigenen Gericht, im Amt oder Ministerium, im Verband oder Unternehmen. Sonst bleibt das eben so eine Sache mit den Frauen, mit der Gleichheit, mit den Grundrechten.

\section{Die Agenda: Grundrechte}

Es zeigt sich, dass die Sache mit der Gleichberechtigung eben nicht „durch“ ist. Journalisten fragen mich das oft. Aber es stimmt nur, wenn die Dinge eng und simpel und flach gefasst werden: Die formelle, flache Version von Gleichheit ist „durch“. Geht es bei der Freiheit um ein Abstraktum, dann ist sie „durch“. Ist die Würde nur das höchste, weit entfernte Gut, dann ist sie „durch“.

Man sollte uns aber mit solchen Fragen weder im Feuilleton noch in der politischen Rede noch im juristischen Kommentar für dumm verkaufen. Es geht in der Sache um mehr als um die Gleichheit der Angeglichenen - und im Grundgesetz ist wie auch in den Menschenrechtskatalogen mehr zu finden. ${ }^{18}$ Man sollte nicht meinen, dass wir jetzt alle zufrieden lächelnd, nett und adrett und endlich auf die Karriere orientiert weiter rackern und nicht meckern, weil endlich alle so einhellig von Gleichheit reden.

Alle reden von Gleichheit? Wir auch. Aber im Ernst. Also auch so wie das Bundesverfassungsgericht.

Vor der Wahl im Richterausschuss des Deutschen Bundestages hat mir ein Abgeordneter eine Frage gestellt: Haben Sie eine Agenda? Er hatte gelesen, dass ich Feministin sei, aus der autonomen Frauenbewegung komme, noch dazu offen lesbisch lebe. Die Antwort lautete: Ja und Nein.

Nein, denn ich halte nichts davon, mit vorgefassten Positionen auf die Suche nach Lösungen für komplexe Probleme zu

16 Zur Rechtslage in der Wissenschaft, wo diese Auffassung besonders gern vertreten wird, vgl. Baer, S., Rechtliche Grundlagen für die Förderung der Gleichstellung in der Wissenschaft, Gutachten für das BMBF 2010; online unter <http://www.bmbf.de/pub/massnahmenfoerderung_chancengleichheit_wissenschaft.pdf> (Zugriff: 12.10.2011).

17 Das BMJ veröffentlicht dazu eine Statistik, derzeit auf dem Stand 2010, unter <www.bmj.de〉. Die Länder führen eigene Statistiken, z.B. NRW unter <http://www.justiz.nrw.de/Gerichte_Behoerden/ zahlen_fakten/statistiken/justizgeschaeftsstatistik $\overline{/}\rangle$. Zahlen über die Richterwahlen finden sich sonst nur verstreut, u.a. <http:// www.jurabilis.de/index.php?/archives/2489-BundesrichterwahlDie-Ergebnisse.html> (Zugriff: jeweils 12.10.2011).

18 Besonders zeitgemäß konkretisiert ist die Charta der Grundrechte der Europäischen Union, unter <http://www.europarl.europa.eu/ charter/pdf/text_de.pdf> (Zugriff: jeweils 12.10.2011). 
gehen, ich hasse Vorurteile und Stereotype und Schubladen. Und mein Leben ist kein politisches Programm.

Und dann aber auch: Ja, ich habe eine Agenda. Meine Agenda ist das Grundgesetz, im Verbund mit den europäischen und internationalen Grund- und Menschenrechten. Über die lässt sich auch als Richterin eingedenk der richterlichen Zurückhaltung gar nicht genug sprechen.

Heute hielt ich das auch für angemessen. Denn das Verfassungsgericht hat die institutionelle Aufgabe, die sich der djb als Nichtregierungsorganisation, als NGO auf die Fahnen geschrieben hat: Den Grundrechten ernsthaft zur Durchsetzung zu verhelfen. Der djb, so heißt es offiziell, zielt auf die „die Fortentwicklung des Rechts auf allen Gebieten“ zur „Ver- wirklichung der Gleichberechtigung und Gleichstellung der Frau in allen gesellschaftlichen Bereichen “. Er fokussiert also. Der djb ist damit Teil einer offenen Gesellschaft der Verfassungsinterpretation, wie es der Rechtswissenschaftler Peter Häberle einmal wenn auch idealistisch formulierte. Heute ist das eine transnationale Zivilgesellschaft, die sich an Grundund Menschenrechten orientiert, den flachen Versionen entgegentritt, sich nicht einlullen lässt, Substanz einfordert. Das Verfassungsgericht ist mit der Aufgabe des Grundrechtsschutzes folglich nicht allein. Um die Grundrechte müssen sich Viele dauernd kümmern.

Dem Deutschen Juristinnenbund und Ihnen allen wünsche ich dabei viel Erfolg.

\section{Gleichberechtigung im Bereich der öffentlichen Hand: Anspruch und Wirklichkeit}

\section{Renate Augstein \\ Ministerialdirigentin, Stellvertretende Leiterin der Abteilung Gleichstellung, Chancengleichheit im Bundesministerium für Fa- milie, Senioren, Frauen und Jugend, Berlin}

Ich freue mich, heute mit Ihnen die Gelegenheit zu haben, die gesetzlichen Instrumente zur Gleichberechtigung im öffentlichen Dienst näher zu betrachten und gemeinsam zu überlegen, in welchen Bereichen sich diese bewährt haben und an welchen Stellen sie weiter geschärft werden müssen. Die öffentliche Hand ist nicht nur durch Artikel 3 Grundgesetz verfassungsrechtlich auf die Gleichberechtigung verpflichtet, sondern ihr kommt auch politisch eine Vorbildfunktion für die gesamte Gesellschaft zu, wenn es um die Herstellung von Gleichberechtigung geht.

Bereits seit 1949 ist in unserem Grundgesetz verankert, dass Männer und Frauen gleichberechtigt sind, und dass niemand wegen seines Geschlechtes benachteiligt oder bevorzugt werden darf. Artikel 3 Grundgesetz wurde später ergänzt um den Staatsauftrag: „Der Staat fördert die tatsächliche Durchsetzung der Gleichberechtigung von Frauen und Männern und wirkt auf die Beseitigung bestehender Nachteile hin". Zur Erfüllung dieses Auftrags wurden verschiedene Instrumente eingesetzt - zum Beispiel das Zweite Gleichberechtigungsgesetz mit dem Frauenförder- und Bundesgremienbesetzungsgesetz 1994, das Bundesgleichstellungsgesetz 2001 und das Allgemeine Gleichbehandlungsgesetz mit der Einrichtung der Antidiskriminierungsstelle des Bundes 2006. Trotzdem haben sich Anspruch und Wirklichkeit in unserem Land nur sehr zögerlich angenähert. Dem von der Bundesregierung 2010 vorgelegten Erfahrungsbericht zum Bundesgleichstellungsgesetz und zum Bundesgremienbesetzungsgesetz lassen sich im Bereich des Anteils von Frauen an den Beschäftigten im öffentlichen Dienst des Bundes und bei der Vereinbarkeit von Familie und Beruf durchaus positive Entwicklungen entnehmen. Der Anteil von Frauen an der Gesamtbeschäftigung in den obersten Bundesbehörden lag 2009 immerhin bei 49 Prozent im Vergleich zu 32 Prozent im Jahr 1986. Familienfreundliche Arbeitsformen wurden in der Bundesverwaltung ausgebaut und werden häufig, inzwischen vermehrt auch von Männern, angenommen.

Gleichzeitig klaffen Anspruch und Wirklichkeit, insbesondere was den Frauenanteil an leitenden Funktionen betrifft, weiterhin auseinander. Frauen sind auf den ersten Führungsebenen in der Privatwirtschaft wie auch im Bereich der öffentlichen Hand nach wie vor selten: Im Jahre 2010 betrug der Anteil von Frauen in den 200 größten deutschen Unternehmen nur 3,2 Prozent bei den Vorständen und 10,6 Prozent bei den Aufsichtsräten. Damit liegt Deutschland im internationalen Vergleich weit zurück. Selbst Schwellenländer wie Brasilien $(6 \%)$ und China $(8 \%)$ weisen einen höheren Frauenanteil in Spitzenfunktionen auf. Bei den Gremien im Einflussbereich des Bundes sieht der Frauenanteil mit knapp 25 Prozent (im Vergleich zu 7\% im Jahr 1991) schon etwas besser aus. Leider gibt es in über zehn Prozent der Gremien immer noch keine einzige Frau. In der Bundesverwaltung liegt der durchschnittliche Anteil von Frauen an Führungspositionen inzwischen bei immerhin 30 Prozent.

Diese Situation ist vor dem Hintergrund der Zukunftsfähigkeit unseres Landes und im Vergleich zu den europäischen Nachbarstaaten weit davon entfernt, zufriedenzustellen. Unter den Vorzeichen des drohenden Führungs- und Fachkräftemangels ist es entscheidend, die nötigen Veränderungsprozesse für eine gleichberechtigte Teilhabe von Frauen in Spitzenfunktionen jetzt anzustoßen. In vielen europäischen Nachbarländern wurde damit längst begonnen und auf EU-Ebene sind bereits unionsweite Maßnahmen geplant. Die Bundesregierung hat sich deshalb die Gleichstellung von Frauen und Männern auf die Fahnen geschrieben. Der Koalitionsvertrag sieht hierzu vor: 